\title{
Modeling of Magnetic Materials
}

\author{
T.G. WOODCOCK ${ }^{1,2}$ \\ 1.-IFW Dresden, Institute of Metallic Materials, PO Box 270116, 01171 Dresden, Germany. \\ 2.—e-mail: t.woodcock@ifw-dresden.de
}

Permanent magnetic materials play a critical role in a wide range of energy applications, for example, electric motors in hybrid vehicles and generators in wind turbines. Materials for these and other applications were brought into focus in JOM, Vol. 64, No. 7, 2012, and were introduced by V. Franco and O. Gutfleisch. The performance of magnetic materials depends not only on the intrinsic properties of the magnetic phase but also on the microstructure and processing conditions. These important aspects were considered in JOM, Vol. 65, No. 7, 2013, and were introduced by M.A. Willard and V. Franco.

To go beyond the state of the art, in terms of both enhancement of performance in a sustainable manner and realization of the next generation of magnets, fundamental knowledge of the behavior of the materials on multiple length scales is required. Characterization of the physical microstructure and magnetic domain structure from $\AA$ to cm length scales is a vital part of this, but often it is difficult to correlate microstructural observations with the magnetic properties. One reason is that local measurements of magnetic properties across a grain or phase boundary are generally not possible in the way that, e.g., the electrical resistivity of such features can be determined. Modeling techniques can be used to connect microstructural observations with magnetic properties and can therefore bring valuable insights into various length scales, which are unobtainable by other techniques. The following three articles focus on different approaches to modeling, operating at very different length scales and report on how the information gained can be used to guide the development of current and future magnetic materials.

In the first article, "Formation of the Face-Centered-Cubic (FCC)-NdO ${ }_{x}$ Phase at $\mathrm{Nd} / \mathrm{Nd}-\mathrm{Fe}-\mathrm{B}$ Interface: A First-Principles Modelling," Chen et al. ${ }^{1}$ use first-principles calculations based on a combination of density functional theory and the cluster expansion method to study the formation mechanism of a metastable face-centered-cubic (fcc) $\mathrm{Nd}$ oxide phase, which commonly appears in Nd-Fe$\mathrm{B}$ sintered magnets. This fcc $\mathrm{Nd}$ oxide may be advantageous in terms of coercivity, and if the formation mechanism can be determined, it may be possible to alter the processing conditions to favor fcc $\mathrm{Nd}$ oxide. In the second article, "Modeling of $\mathrm{Nd}$ Oxide Grain Boundary Phases in Nd-Fe-B Sintered Magnets," Hrkac et al. ${ }^{2}$ use atomistic and micromagnetic modeling techniques based on electron microscopy results to investigate the magnetic properties of interfaces between the $\mathrm{Nd}_{2} \mathrm{Fe}_{14} \mathrm{~B}$ phase and various $\mathrm{Nd}$-rich phases. These features were incorporated into finite element micromagnetic models that allowed realistic predictions of coercivity as a function of grain size to be calculated for a two-grain system. In the final article, "Geometry Dependence of Magnetization Reversal in Nanocomposite Alloys," Skomski et al. ${ }^{3}$ consider next-generation hard magnets in the form of exchange-coupled nanocomposites. The authors explore the optimum microstructure for nanocomposite magnets by finding solutions to the nucleation field equation for various geometries of the hard and soft magnetic phases. In addition, the crucial influence of magnetostatic interactions between the phases is considered using the two-spin model for various geometries.

\section{REFERENCES}

1. Y. Chen, A. Saengdeejing, M. Matsuura, and S. Sugimoto, JOM 66 (2014). doi:10.1007/s11837-014-1004-1.

2. G. Hrkac, K. Butler, T.G. Woodcock, L. Saharan, T. Schrefl, and O. Gutfleisch, JOM 66 (2014). doi:10.1007/s11837-0140980-5.

3. R. Skomski, P. Manchanda, I. Takeuchi, and J. Cui, JOM 66 (2014). doi:10.1007/s11837-014-1005.0.

Thomas Woodcock is the guest editor for the Magnetic Materials Committee of the TMS Functional Materials Division (formerly EMPMD), and coordinator of the topic Modeling of Magnetic Materials in this issue. 